\title{
Perspective
}

PERSPECTIVE Actualité en histoire de l'art

$1 \mid 2020$

Japon

\section{Art et Préhistoire au Japon : les Jōmon}

Une discussion entre Jean-Paul Demoule et Inada Takashi, menée par Laurent Nespoulous

Jean-Paul Demoule, Takashi Inada et Laurent Nespoulous

Traducteur : Laurent Nespoulous

(2) OpenEdition

Journals

Édition électronique

URL : http://journals.openedition.org/perspective/17408

DOI : $10.4000 /$ perspective. 17408

ISSN : 2269-7721

Éditeur

Institut national d'histoire de l'art

Édition imprimée

Date de publication : 5 juin 2020

Pagination : $23-40$

ISBN : 978-2-917902-89-9

ISSN : $1777-7852$

\section{Référence électronique}

Jean-Paul Demoule, Takashi Inada et Laurent Nespoulous, «Art et Préhistoire au Japon : les Jōmon », Perspective [En ligne], 1 | 2020, mis en ligne le 30 décembre 2020, consulté le 24 janvier 2021. URL : http://journals.openedition.org/perspective/17408; DOI : https://doi.org/10.4000/perspective. 17408 


\title{
Art et Préhistoire au Japon : les Jōmon
}

\author{
Une discussion entre Jean-Paul Demoule et Inada Takashi, \\ menée par Laurent Nespoulous
}

Comme je le soulignais il y a maintenant un peu plus d'un an lors de la dernière exposition Jōmon : Naissance de l'art dans le Japon préhistorique à Paris (octobre-novembre 2018), la Période Jōmon (13000 à 800 avant notre ère) occupe une place singulière, et ce pour plusieurs raisons ${ }^{1}$. Elle n'est certes pas constitutive de l'histoire du Japon au sens strict : ce dernier nom ne surgira pas avant la seconde moitié du VII ${ }^{\mathrm{e}}$ siècle de notre ère, et ce serait un anachronisme que de toujours tout ramener à une éventuelle japonité des phénomènes : serait-il pertinent de faire commencer l'histoire de France avec la grotte Chauvet ? Cette période archéologique n'en occupe pourtant pas moins une place incontournable à la fois dans l'histoire des sociétés préhistoriques de l'archipel, mais également dans la construction d'un nouveau rapport au passé depuis l'époque d'Edo, c'est-à-dire depuis le XVII ${ }^{\mathrm{e}}$ siècle, jusqu'à nos jours.

C'est en effet grâce à la découverte de poteries préhistoriques datant de ce que l'on sait désormais avoir été la fin de la période Jōmon, dans le Nord-Est de l'archipel, que certains penseurs et collectionneurs du Japon des $\mathrm{XVII}^{\mathrm{e}}$ et $\mathrm{XVIII}^{\mathrm{e}}$ siècles prirent conscience de l'existence d'un temps plus profond que ce qu'une approche du passé par les textes pouvait laisser saisir.

Cette poterie, d'abord désignée du nom de ses premiers gisements de découverte - poterie de Kamegaoka -, est rebaptisée plus tard, à la fin des années 1870, par le naturaliste américain Edward S. Morse (1838-1925), du nom de cord marked pottery. C'est cette expression qui donne, en traduction japonaise enfin le terme jōmon (littéralement " décor à la corde ") : une poterie dont le décor se caractérise par l'impression de motifs réalisés au moyen de cordelettes tressées, pressées sur la pâte des productions en terre avant cuisson. Morse, spécialiste des mollusques et des brachiopodes, enseignant à Harvard University, est le premier professeur à enseigner la zoologie au Japon, à l'Université impériale de Tōkyō. Ce sont ses travaux sur les brachiopodes, justement, qui le mènent au Japon en 1877, où il fouille l'amas coquillier d'Ōmori. Ces amas, monticules de rejets formés par l'activité humaine de récolte et de consommation de coquillages, très fréquents au Japon, sont également riches en objets fabriqués de la main de l'homme. De ce fait, Morse, en plus d'étudier les amoncellements de mollusques anciens, fait la découverte d'artefacts et met au jour, à son tour, la Préhistoire de l'archipel. 
Morse ne se situe alors pas du tout sur le terrain de l'art quand il désigne ses trouvailles, à la différence de ses prédécesseurs de l'époque d'Edo, pour qui ces poteries sont des objets de collection depuis longtemps déjà.

Concernant l'archéologie de la période Jōmon, voire même l'archéologie " tout court ", la littérature scientifique japonaise tend depuis bien des décennies à présenter Morse comme son père fondateur le plus illustre. Quant à la première mise en ordre scientifique de la période Jōmon elle-même, elle est attribuée très généralement à un archéologue incontournable de l'après-guerre, Yamanouchi Sugao (1902-1970). Ceci était d'ailleurs rappelé par Shinagawa Yoshiya dans le catalogue de l'exposition parisienne de l'automne 20182. Les questions de "paternité " comportent sans doute une bonne part de vanité, mais dans les cas de Morse aussi bien que de Yamanouchi, ne retenir que ces deux noms relève d'une vision bien étroite de l'histoire des sciences. Tout d'abord parce que Morse n'a pas vraiment eu de postérité ni poussé bien loin ses investigations préhistoriques (il n'a formé aucun archéologue et n'était pas venu au Japon pour cela), et ensuite parce que Yamanouchi Sugao ne fut pas le premier à tenter une première mise en forme de la Préhistoire japonaise. Et c'est en cela que la période Jōmon se singularise encore davantage !

À la fin des années 1920 et au tout début des années 1930, c'est un jeune archéologue japonais, de la même génération que Yamanouchi Sugao, Nakaya Jiujirō (1902-1936), qui devient l'émissaire en Europe de l'archéologie préhistorique naissante du Japon. Ses premières publications en japonais datent de 1927, et il est l'auteur du premier manuel japonais de préhistoire (1929), d'ailleurs réédité encore en $1943^{3}$. En ce sens, il est l'inventeur de la période Jōmon, qu'il présente en Europe comme un équivalent japonais du Néolithique. Venu à Paris de l'université impériale de Tōkyō, afin d'étudier auprès de l'ethnologue Paul Rivet (1876-1958, directeur du musée d'ethnographie du Trocadéro), c'est lui qui introduit pour la première fois dans le détail en Europe les productions de la période Jōmon ${ }^{4}$. De la Revue des Arts asiatiques à la revue Documents, en prenant pour sujet les poteries à bec verseur de la fin du Jōmon et les figurines en terre cuite, les dogū, Nakaya est le véritable premier véhicule d'une connaissance disciplinaire de la Préhistoire japonaise en Europe. Ses travaux posent les premières classifications des productions Jōmon sur la base des formes, des décors et de leur répartition sur le territoire japonais.

Première culture préhistorique découverte à révéler un temps profond de l'histoire humaine au Japon, première période à faire l'objet d'une diffusion précoce dans le monde occidental, première étape d'une histoire de l'art propre à l'archipel : Jōmon est tout cela, en plus d'être aujourd'hui une période archéologique de plus de 10000 ans. [Laurent Nespoulous]

- Laurent Nespoulous. Ceci étant posé, je souhaiterais revenir sur le traitement même de l'art dit Jōmon au Japon et vous poser à chacun une première question. Jōmon offre une grande variété d'expressions régionales et chronologiques, mais il est vrai que chaque fois, au moins depuis le Jōmon ancien (de 5000 à 3500 avant notre ère), dans chaque ensemble chrono-culturel, semble exister une réelle "grammaire » des décors employés, décors et formes plastiques ne faisant d'ailleurs pas du tout nécessairement appel aux décors en impression de corde. Il est donc clair qu'une codification poussée existait, de même qu'existaient des exécutants plus ou moins doués. Est-ce que l'on a raison, de nos jours, de parler d'art ? Est-ce que ce n'est pas une manière un peu « facile » de cataloguer un phénomène, un langage, qui nous reste assez inaccessible? 
- Jean-Paul Demoule. La notion d'« art », appliquée à des sociétés traditionnelles, pose effectivement problème, puisque dans ces sociétés, ce que nous appelons « art » n'est pas séparé des autres productions matérielles, contrairement à ce qui se passe dans nos propres sociétés contemporaines. L'anthropologue Franz Boas fut sans doute le premier à traiter cette question dans Primitive Art ${ }^{5}$, qui n'a d'ailleurs été traduit en français qu'en 20036. II se fondait plus particulièrement sur l'étude des productions des Amérindiens de la côte nordouest du Pacifique, de l'autre côté de cet océan, chez lesquels s'étaient développées des sociétés de chasseurs-cueilleurs sédentaires et hiérarchisées, sans doute les meilleurs points de comparaison ethnographique avec les sociétés Jōmon. Boas considérait notamment que l'on pouvait parler $d^{\prime}$ '« art » à partir d'un certain degré d'investissement technique sur des objets socialement valorisés, qu'ils soient de petite taille (masques, boîtes ornées, vanneries, etc.) ou bien immobiliers, comme les célèbres grands mâts totems de cette région.

- Laurent Nespoulous. Encore faudrait-il être toujours capable de saisir ce qui relevait de fait d'un « investissement technique » et ce qui n'était pas utilitaire. Si l'on suit cette piste, qu'est-ce que nous laisse percevoir l'archéologie?

- Jean-Paul Demoule. Les premiers témoignages d'activités matérielles non utilitaires peuvent remonter à l'homo erectus qui a tracé il y a 500000 ans sur le site de Trinil, dans l'île indonésienne de Java, des zigzags méthodiques sur une valve de coquillage. Mais plus généralement, cette espèce humaine, la première à être sortie d'Afrique il y a quelque deux millions d'années, avait entrepris de réaliser des bifaces, outils à trancher parfaitement symétriques, beaucoup plus soignés et réguliers qu'il n'était nécessaire pour leur fonction. Aussi le préhistorien André Leroi-Gourhan a-t-il supposé que les processus d'hominisation avaient pu en particulier s'accompagner d'un développement des sentiments esthétiques. On doit également à I'homme de Néandertal quelques tracés non figuratifs, ainsi que les premières parures pour le corps.

C'est seulement au cours de l'évolution d'homo sapiens, il y a 40000 ans environ, que les manifestations esthétiques prennent véritablement leur essor. Trois formes peuvent en être distinguées. La première est celle de signes abstraits, dans la lignée des premiers zigzags, signes dont il existe une typologie stable sur les murs des grottes, indice qu'ils signifiaient quelque chose - ce qui mène, bien plus tard, à l'écriture. La seconde est celle d'une esthétique des objets quotidiens valorisés, telle que l'étudia Boas, et qui s'enracine, comme la première, dans les traditions antérieures, cette fois des bifaces inutilement symétriques; elle est manifeste, par exemple, dans l'ornementation des propulseurs ou des bâtons percés de l'époque magdalénienne. La troisième est celle des représentations proprement dites, animales pour l'essentiel, mais également humaines et, dans ce cas, féminines en grande majorité.

- Laurent Nespoulous. D'une manière générale, les formes les plus " plastiques » d'art semblent particulièrement faire leur apparition dans le contexte du dernier maximum glaciaire, dans l'univers eurasiatique. On mentionne souvent à cet égard le Gravettien, et ce que l'on pourrait considérer comme des variantes proches de ce dernier plus encore vers l'ouest, comme étant déterminé par un « kit » de caractéristiques dont les figurines font partie.

- Jean-Paul Demoule. C'est au moment où s'épanouissait la civilisation magdalénienne dans l'extrême Ouest de l'Eurasie, qu'à son extrémité orientale, en Chine (à partir de 18000 ans avant notre ère) et au Japon (à partir de 14000 ans avant notre ère), d'autres sociétés de chasseurs-cueilleurs inventèrent les premières poteries - la terre cuite étant déjà attestée 


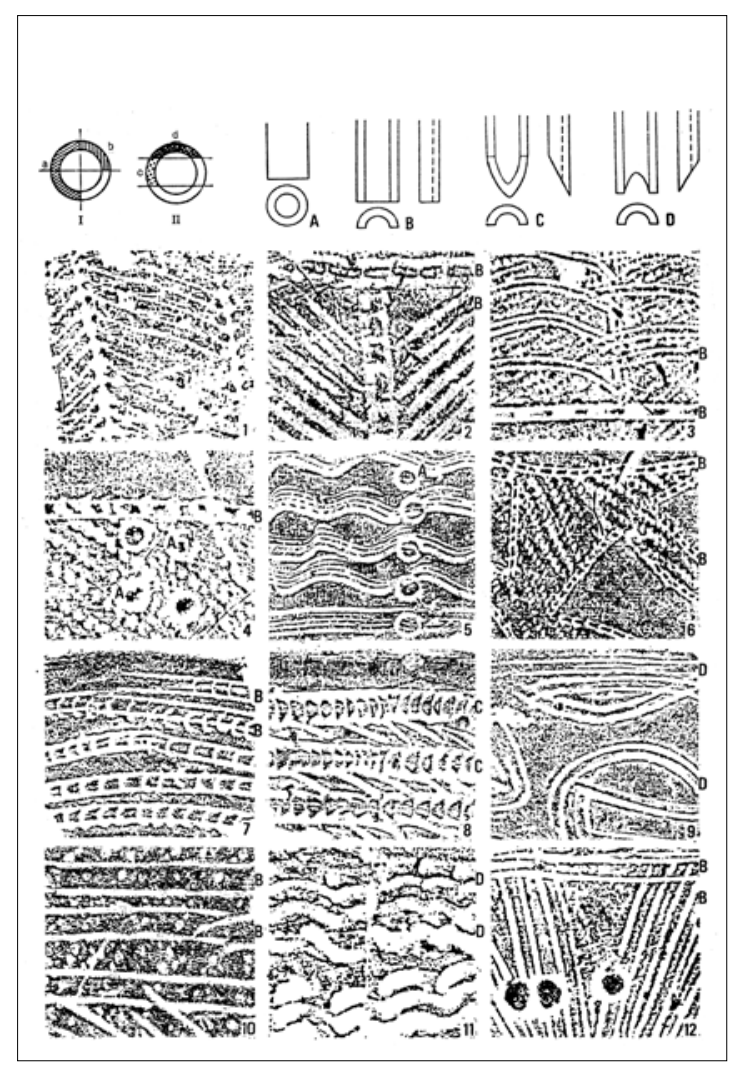

1. Différents motifs réalisés avec un bambou fendu, publié dans Kani Michihiro,

「施文原体の変遷、竹管文、分類と展開」,

dans Kikan kōkogaku『季刊考古学』， $n^{\circ}$ 17， 1986, p. $77-80$, p. 78 dès 25000 ans avant notre ère dans la civilisation gravettienne de l'Europe centrale, pour de petites figurines. Ces premières poteries ne sont pas, ou très peu, ornées. Mais elles vont l'être de plus en plus. Ailleurs, en Sibérie, en Asie centrale ou en Amazonie, des chasseurs-cueilleurs créent également des poteries, liées en général à un mode de vie sédentaire, qui évite d'avoir à transporter ces objets fragiles.

- Laurent Nespoulous. Cela revient à dire que I'invention de la poterie, puis son utilisation comme support à des fins " artistiques", prend d'abord son essor dans des sociétés non agraires, dans le contexte du Tardiglaciaire. La poterie Jōmon, de ce point de vue, est, me semble-t-il, particulièrement intéressante dans la mesure où elle fait le lien entre les sociétés de la fin de la dernière glaciation et celles qui continuent à évoluer dans un nouveau contexte, celui du réchauffement climatique de l'Holocène.

- Inada Takashi. Les décors de la poterie Jōmon sont riches d'une grande diversité : grande variété de motifs, mais également de formes. Si à cela on ajoute maintenant la diversité régionale et chronologique, le tableau se complique encore davantage et il devient pour le coup difficile de mettre le doigt sur ce que serait, en fait, la poterie Jōmon en tant qu'ensemble cohérent.

Il faut bien comprendre que la poterie Jōmon a d'abord été étudiée selon une approche typochronologique, qui a consisté à fixer le cadre chronologique et géographique des différents types céramiques, et d'établir ensuite des correspondances avec tous les autres éléments de la culture matérielle - qu'il s'agisse des habitations, des tombes, de l'industrie lithique ou osseuse -, le tout dans l'objectif d'appréhender cette culture. De ce point de vue, la grande complexité de la poterie Jōmon est un beau cadeau fait à l'exercice de la typo-chronologie ! De nos jours, sur la grosse dizaine de milliers d'années que représente la période Jōmon partout dans l'archipel, nous pouvons compter pas moins d'environ 80 styles de poterie, lesquels font à leur tour l'objet de subdivisions en plusieurs centaines de types ${ }^{7}$. Autrement dit, la typo-chronologie a en elle-même pour caractéristique de fabriquer un cadre spatial et chronologique, et cette démarche conduit sans doute en elle-même à une telle complexité. Par conséquent, lorsque l'on se pose la question du sens de cette très grande diversité de formes, et de motifs, il est nécessaire de reformuler entièrement les résultats de la typo-chronologie en leur appliquant un regard qui est cette fois-ci celui de I'historien.

Yamanouchi Sugao a été le grand « leader » de l'étude typo-chronologique de la poterie Jōmon. Pendant longtemps le terme de Jōmon renvoyait à l'idée de l'impression des fibres de motifs textiles obtenus par tissage ou tricotage. C'est Yamanouchi qui a démontré, dans les années 1930, que ces motifs " en impression de cordes » étaient obtenus en faisant 
rouler des cordelettes sur la terre crue (motifs en impression de cordes roulées). Par la suite, il a démontré, par l'expérimentation, que la grande diversité des motifs imprimés de cordes (jōmon) pouvait être obtenue par les différentes manières de tresser ces cordelettes, d'associer différents tressages ensemble, et de les enrouler autour de bâtonnets pour les faire rouler de différentes façons sur la pâte. Ce travail a constitué sa thèse de doctorat en $1961^{8}$.

- Laurent Nespoulous. C'est par là que l'on commence quand on veut expliquer au grand public ce que veut dire Jōmon. Mais cela se complique ensuite, quand il faut souligner que la période ne se limite en réalité pas à cette méthode de décoration.

- Inada Takashi. Il est en effet bien connu des spécialistes que la poterie Jōmon ne se limite pas à des décors en impression de cordes, mais également de quelques sortes de coquilles de bivalves, ou en incisions faites au moyen de bambou, qu'elle peut porter des marques volontaires de doigts et d'ongles, et qu'elle fait aussi un usage plastique marqué des colombins de terre. Je désigne toutes ces poteries à motifs dérivant de façon immédiatement reconnaissable de la forme de l'« ustensile » utilisé, " poterie à motifs en empreinte » (semongukeitai).

Par ailleurs, lorsqu'un motif est obtenu par l'agencement de ces empreintes sur la surface de la pâte, cela permet toujours de reconstituer les gestes de l'auteur (position de travail, direction). Comme dans ce cas le motif final ne résulte pas tant de la forme de l'empreinte en elle-même que des motifs que plusieurs empreintes forment ensemble, je parle alors de « motifs directionnels » (hōikeitai). Par exemple, pour un motif en impression de corde sur une forme cylindrique, on placera la cordelette verticalement sur la pâte, et on la fera rouler à l'horizontale, faisait ainsi apparaître la direction du motif. Yamanouchi, en faisant la découverte de l'usage de la cordelette comme outil de décor, a donc dans le même temps découvert la direction du motif.

Il en va de même pour les autres types d'empreintes: que ce soit avec les ongles ou un bambou fendu, on finira par obtenir des agencements en lignes droites ou courbes. Avec le bambou fendu, si l'on se met à tracer des parallèles, on obtiendra des motifs toujours plus complexes (fig. 1). Si I'ustensile est une fine baguette, alors la direction du motif sera entièrement libre. On obtient alors un « motif linéaire en incision » (chinsenmon). Ici, la forme entière de l'outil est perdue dans le motif, et l'on n'en conserve que l'épaisseur.

On peut donc dire d'abord que ces deux types de réalisation des motifs sont les deux faces inséparables de la production du décor de la poterie et que ce dernier relève d'un motif qui sublime soit la forme même de l'outil employé (comme le motif à cordelettes), soit la direction du geste de l'auteur (comme le motif linéaire incisé).

$\mathrm{Si}$, maintenant, on se penche sur l'historicité de ces deux types de motifs, voilà ce que l'on peut

2. Vase à application de matière " en arachide ", phase initiale, Sasebo (département de Nagasaki), abris de Senpukuji, Comité éducatif de Sasebo.

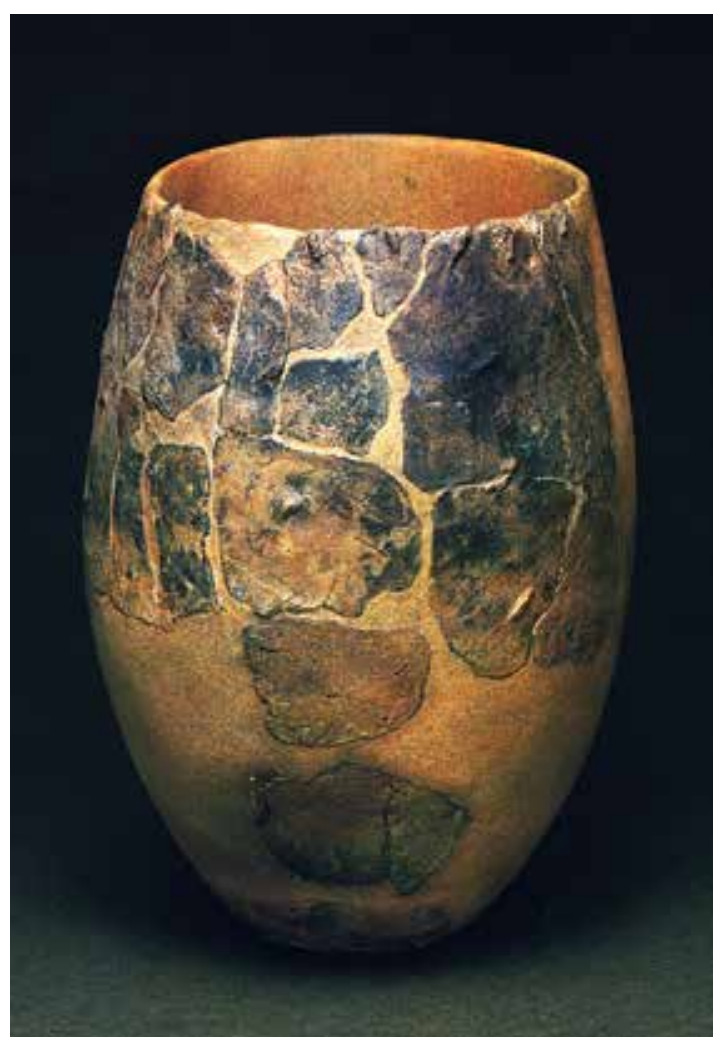



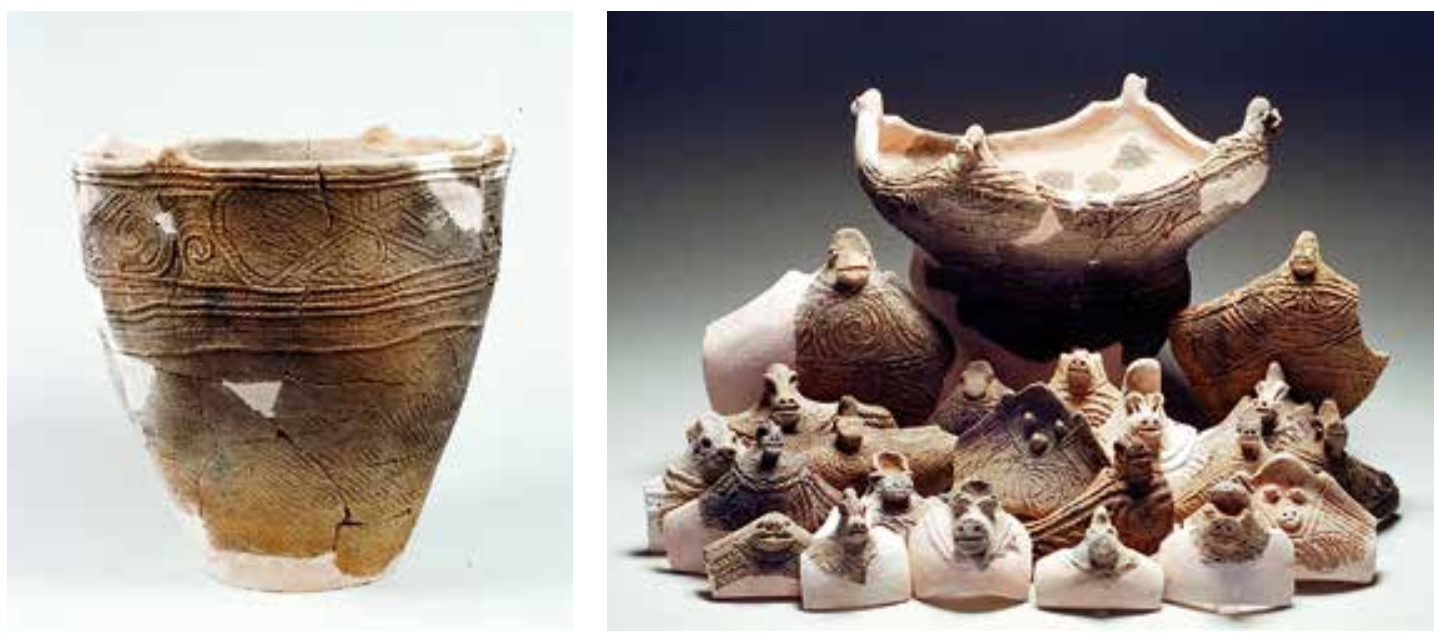

3. Motifs réalisés au bambou, phase ancienne, Annaka (département de Gunma), site de Nakanoya Matsubara, Comité éducatif de Annaka.

4. Poteries à anses en "face de bête ", phase ancienne, Annaka (département de Gunma), site de Nakanoya Matsubara, Comité éducatif d'Annaka.

5. Poterie "flammiforme », phase moyenne, Tsunan (département de Niigata), site de Dōjitte, Comité éducatif de Tsunan. brièvement en dire. La poterie fait son apparition dans l'archipel au moins vers 14000 avant notre ère, moment qui peut être qualifié de phase initiale de la période Jōmon, ou proto-Jōmon. Les plus anciens exemplaires de cette poterie proviennent du site d'Ōdaiyamamoto (département d'Aomori), et consistent en quelques tessons, non décorés. Le stade suivant se caractérise par l'apparition du « motif linéaire enroulé » (ryūsenmon, lignes enroulées rappelant les empreintes digitales) composé de fins rubans et de pièces de terre en forme d'arachide appliqués sur la poterie (fig. 2). Ensuite apparaissent les premiers motifs créés à partir d'impression

d'ongles. Jusque-là, les décors sont réalisés principalement à la main. C'est à la toute fin de cette première phase du Jōmon que les motifs par impression de corde et la technique de la cordelette roulée font leur apparition.

Le Jōmon archaïque (9500-5000 avant notre ère), et le Jōmon ancien (5000-3500 avant notre ère) sont les phases où l'on voit se développer cette dernière technique et se multiplier l'usage d'objets divers afin de produire des motifs par impression. Le Jōmon ancien se caractérise par le développement de motifs obtenus grâce au bambou (fig. 3 et 4).

Le Jōmon moyen (3500-2500 avant notre ère) est un moment où l'on voit se développer un art plastique particulièrement exubérant, avec le recours à des applications de matière pour les décors, ainsi qu'au façonnage de grandes anses placées au niveau de l'ouverture des récipients. C'est cette expression de la poterie Jōmon qui est internationalement la plus connue, alors qu'elle ne constitue - comme le célèbre motif plastique en forme de flamme (kaendoki, fig. 5) - qu'une expression particulière du Jōmon de la région du Chūbu (vaste région autour de Nagano) de l'archipel. Le Jōmon moyen se caractérise par des décors mélangeant à la fois des applications de fins rubans de matière et des motifs linéaires incisés.

Les céramiques des Jōmon récent (2500-1300 avant notre ère) et final (1300-600 avant notre ère) sont principalement marquées par des motifs directionnels formés d'incisions de lignes. De ces incisions découlent deux types de motif, les «motifs délimitants » (kukakumon) et les « motifs autonomes » (tan'imon). Les premiers consistent en lignes incisées enceignant la poterie. En répétant l'opération, on peut ainsi isoler différentes zones à décorer. Par exemple, si une ligne dans la partie supérieure du récipient et une autre dans sa partie inférieure 


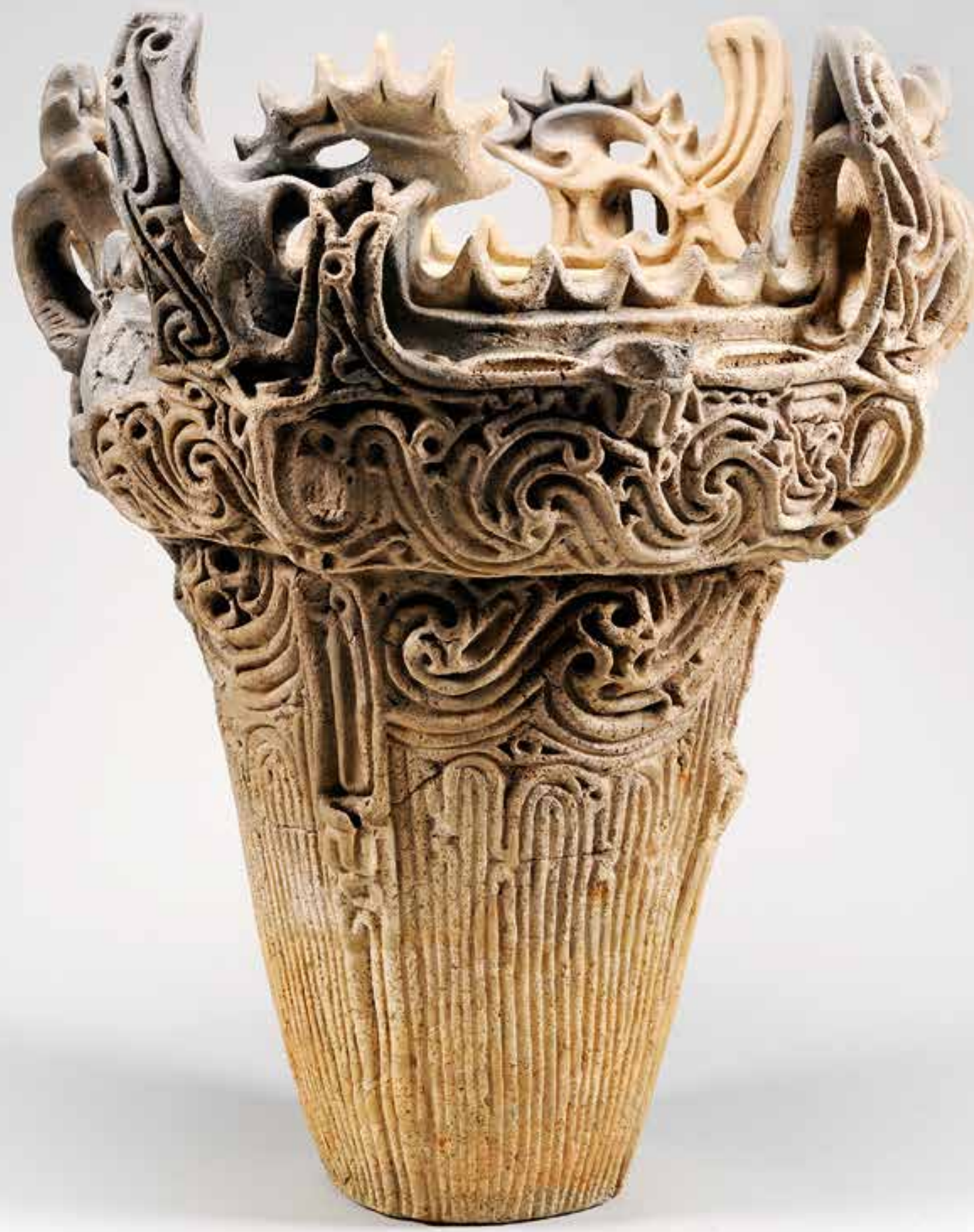



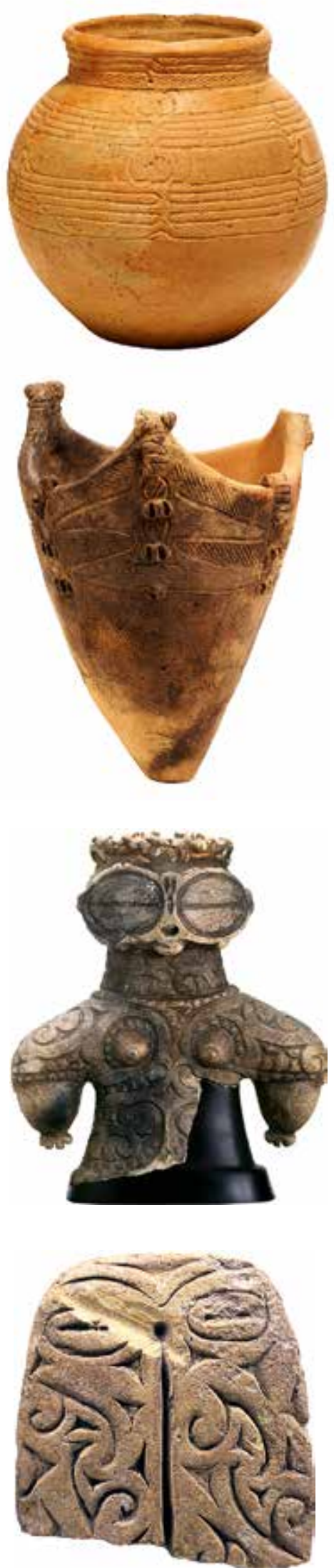

sont marquées, en les recoupant par des lignes perpendiculaires on produira des cellules rectangulaires, et si l'on opte pour des diagonales, des cellules triangulaires (fig. 6 et 7 ).

Les seconds sont des motifs en arrangement préétabli, qui constituent en eux-mêmes un motif (irikumimon, sansamon). Ces motifs ont donc une forme élaborée, mais qui ne structure pas le décor. C'est la raison pour laquelle ils se trouvent associés aux motifs délimitant, à l'intérieur d'espaces formés par ces derniers, ou entre ces derniers. En l'absence de cellules vis-à-vis desquelles les placer, les motifs autonomes sont simplement répétés les uns à côté des autres, de sorte à enceindre, à leur façon, la poterie (fig. 8).

C'est durant cette phase du Jōmon que l'on voit également se développer et se répandre la technique de polissage, de lustrage des productions (surikeshi jōmon, fig. 6, 7, 8 et 9). Entre les incisions de lignes, on imprime un motif de cordelette, qui vient, par polissage et lustrage, mettre davantage en valeur le relief des incisions. Le motif incisé occupe donc le rôle central, et le motif imprimé a plus pour fonction de le faire valoir.

Les motifs autonomes qui se généralisent alors échappent largement au domaine de la terre cuite (poterie et dogū, fig. 9) et on les retrouve gravés sur des plaquettes de pierre (ganban, fig. 10), mais aussi sur des objets en bois dont la fonction est inconnue (herajō mokki, littéralement, " objet en bois en forme de spatule ", fig. 11). Les motifs de types directionnels, extrêmement bien maîtrisés, franchissent donc la barrière de leur support d'origine. Ce n'est pas le cas des motifs obtenus par impression, qui ne semblent pouvoir se trouver vraiment sublimés que sur des supports malléables.

- Jean-Paul Demoule. Curieusement, lorsque le Néolithique apparaît vers 9500 avant notre ère au Proche-Orient avec l'agriculture sédentaire, la poterie reste inconnue dans cette région et n'y apparaît que deux millénaires plus tard. On trouve donc des récipients en pierre ou bien, plus rarement, en matières périssables (bois, cuir, vannerie). Avec la généralisation progressive de l'agriculture, le boom démographique qu'elle entraîne et l'absorption des chasseurs-cueilleurs par les agriculteurs, les poteries deviennent partout l'un des objets indispensables de ces nouvelles sociétés. Faciles à fabriquer en nombre, elles servent aussi bien au stockage qu'à la cuisson ou

6. Vase à "motifs délimitants" et à " motifs autonomes ", phase récente, Sakura (département de Chiba), site de Miyauchi idosaku, Centre pour les biens culturels de l'intercommunalité d'Inbagun.

7. Vase à motifs autonomes, phase finale, Sakura (département de Chiba), site de Miyauchi idosaku, Centre pour les biens culturels de l'intercommunalité d'Inbagun.
9. Dogū à décor entrelacé (sansamon), phase finale, Hachinohe (département d'Aomori), site de Korekawa-Nakai, Comité éducatif de la ville de Hachinohe.

10. Plaque de pierre à décor entrelacé (sansamon), Hachinohe (département d'Aomori), site de Korekawa-Nakai, Comité éducatif de la ville de Hachinohe. 


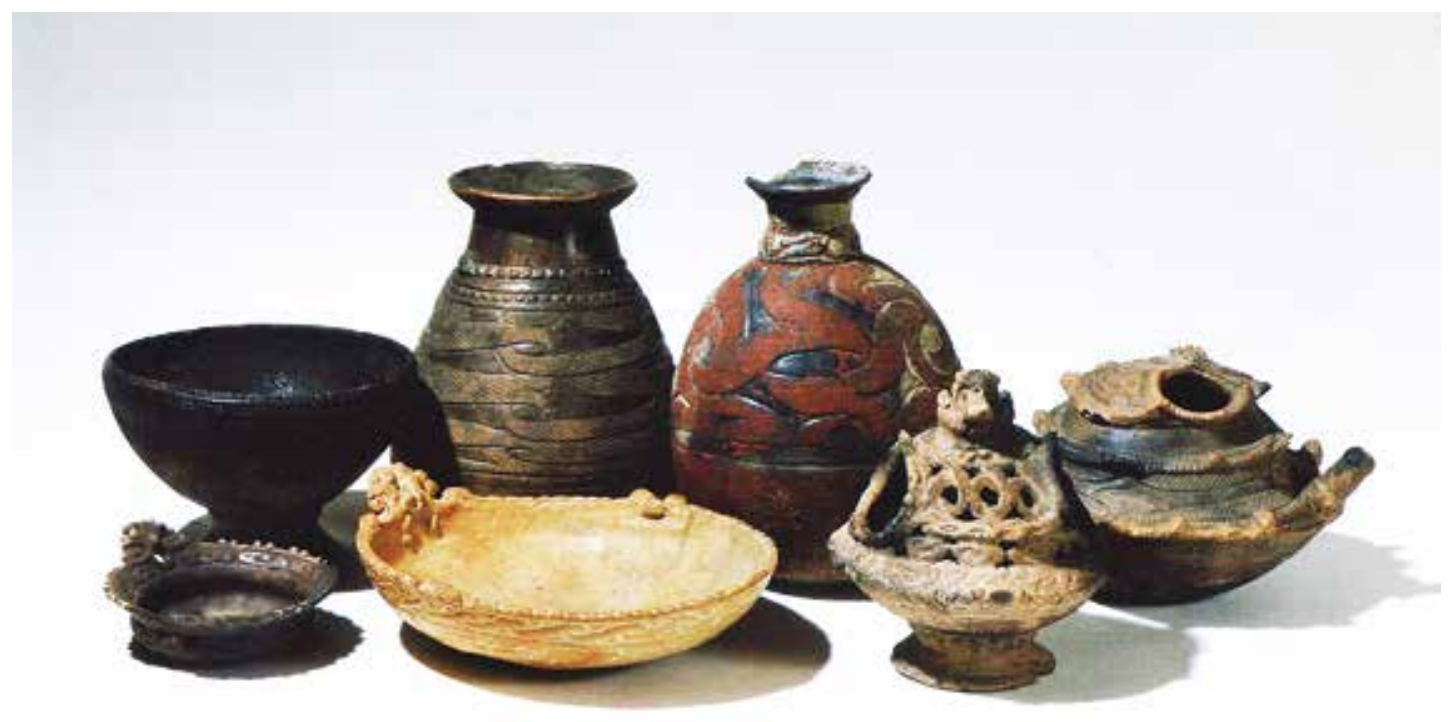

à la consommation des aliments. Les formes des poteries peuvent répondre en partie à des contraintes fonctionnelles, 8. De gauche à droite, bol, plats, vases, poterie en forme de brûle-parfum, poterie à bec verseur, phase finale, Hachinohe (département d'Aomori), site avec une partie supérieure resserrée s'il s'agit de stocker et de verser des liquides, ou au contraire largement ouvertes pour la consommation des aliments, encore que ces de Korekawa-Nakai, Comité éducatif de la ville de Hachinohe. contraintes puissent être relativement souples, et certains récipients multifonctionnels. En revanche les décors sont évidemment libres de toute contrainte, sinon celles des normes culturelles et des capacités techniques. Ces récipients offrent donc un nouveau support à l'expression esthétique et l'on retrouve, de par le monde, trois principales techniques d'ornementation : soit des motifs peints, soit des motifs gravés sur la pâte fraîche avant cuisson (les archéologues parlent de décors " incisés »), soit enfin des décors par impression d'objets divers - des doigts, des ongles, des poinçons, des peignes, voire des objets plus élaborés comme dans le Jōmon japonais. À cela peuvent s'ajouter des ornements plastiques, ajoutés, ce dont témoignent en particulier certains vases très spectaculaires du Jōmon moyen.

- Laurent Nespoulous. Est-ce qu'il est possible de donner un sens historique à ce phénomène dans son ensemble et à toute sa diversité ?

- Inada Takashi. Si l'on divise la période Jōmon en deux (Jōmon initial, archaïque, ancien d'une part, et moyen, récent et final d'autre part) il est possible de percevoir un certain nombre d'éléments qui nous renseignent sur la « trajectoire » empruntée par les décors de la poterie.

Durant la première moitié du Jōmon, les motifs par impression se développent et se diversifient ; on les retrouve, dans la seconde moitié de la période, sublimés sous la forme des motifs directionnels par incision de lignes.

Les motifs linéaires incisés sont certes présents dans la première moitié du Jōmon, mais ils sont en compétition avec les motifs imprimés à l'aide d'autres moyens, et ils constituent parmi cet ensemble de décors les motifs les plus simples et pauvres. Dans la mesure où la forme caractéristique de l'objet utilisé pour pratiquer l'incision disparaît sous le trait tracé, on y perd même la notion de motif imprimé. 
Ces mêmes motifs linéaires, qui offrent en eux-mêmes une plus grande liberté de composition que ceux obtenus par impression, sont exploités dans tout leur potentiel au cours de la seconde moitié de la période Jōmon. Les motifs principaux sont alors essentiellement de type directionnel, et les motifs par impression ont désormais un rôle auxiliaire alors que ceux qui structurent la « lecture » du décor se répandent sur d'autres supports.

Les décors de la poterie Jōmon ne prennent pas modèle sur des motifs ou des représentations picturales déjà existantes, et semblent bien s'être développés en même temps que la production de poterie elle-même dans l'archipel. Parmi ces motifs, les plus aboutis franchirent donc même les limites de leur support en terre pour venir orner d'autres objets, devenant par là même un mode d'expression en soi, qui nous permet d'apercevoir un univers spirituel d'un haut niveau d'abstraction.

Ces motifs, nés sur un support avant tout utilitaire, se sont ensuite développés au point de venir, par exemple par leur exubérance plastique, gêner la fonction utilitaire première des récipients. Une trop grande proximité entre l'orbe de la spiritualité et celle de I'utile nous rend compte de ce que nos yeux actuels pourraient comprendre comme une forme d'immaturité ou de primitivité des gestes de production, et peut constituer un frein à la libération de l'une par rapport à l'autre.

Inciser des lignes ou pratiquer un polissage des motifs imprimés ne sont pas des techniques d'une grande complexité et c'est pourquoi on les retrouve dès la première moitié de la période Jōmon. Ce qui est important c'est le fait que ces motifs, d'abord éparpillés ou relativement limités spatialement et chronologiquement, finissent par se généraliser durant la seconde moitié du Jōmon. L'incision des motifs linéaires a tendance, dans la plupart des cas, à résulter d'un griffonnage de la terre crue durant la première moitié de la période, puis elle consiste à graver soigneusement en surface durant la seconde moitié de la période.

- Jean-Paul Demoule. Dans une grande partie du Proche-Orient, c'est au début la peinture qui a prévalu, et de là dans l'ensemble de la péninsule Balkanique, I'Italie et le Sud de l'Europe centrale ; on la trouve aussi dans les phases récentes du Néolithique chinois, ou aussi bien en Amérique centrale et méridionale. Les décors incisés se sont développés dans l'Europe centrale puis occidentale (culture dite de la Céramique linéaire ou Rubané) et cette tradition s'y est poursuivie aux âges du bronze et du fer - de même qu'ils prennent le dessus dans les phases récentes du Jōmon, comme le décrit très bien Inada Takashi. Les décors imprimés ont concerné une petite partie du Proche-Orient (Byblos) et le long des côtes nord de la Méditerranée (culture dite impresso-cardiale). Mais ils ont été également majoritaires en Asie centrale, en Ukraine, en Russie et en Sibérie ; c'est donc sans surprise qu'on les retrouve dans la péninsule japonaise. Il semble en effet que, au-delà des particularités culturelles régionales, se soient imposées de grandes zones de préférences stylistiques.

Il faut aussi remarquer que, dans ces sociétés villageoises, l'ornementation est en très grande majorité géométrique et symétrique. Elle n'est que très exceptionnellement figurative, contrairement à ce qui se passe bien plus tard dans les sociétés étatiques, comme sur les vases de la Grèce antique ou sur la porcelaine chinoise. On a parfois mis en rapport ce remplissage strict de l'espace à orner avec la prise de contrôle de la nature que représente l'agriculture, avec ses défrichements, ses pacages et ses champs cultivés. On pourrait objecter alors que les sociétés du Jōmon ne sont pas agricoles, et que l'alimentation provient pour l'essentiel de la chasse, de la pêche et de la cueillette. Pourtant, au fur et à mesure que ces sociétés évoluent dans les quelque treize ou quatorze millénaires qu'elles ont duré, elles ont à la fois gagné en complexité dans leur organisation, mais aussi dans leur contrôle de la nature. Les preuves de hiérarchies sociales, au sein de grands villages permanents, ne manquent pas. Elles ont également exercé des formes de sylviculture sur les chênes et les marronniers pour 
produire glands et marrons, en favorisant ces espèces forestières au détriment des autres. Elles ont introduit dans des îles des sangliers et des cerfs, créant ainsi des sortes de réserves de chasse. Enfin, la pratique d'une petite horticulture d'appoint, attestée chez certains chasseurs-cueilleurs d'autres régions du monde, a également pu être mise en évidence.

De fait, ce terme de " Jōmon » peut faire croire à une unité et à une homogénéité culturelles fortes et inchangées, alors qu'en réalité il englobe une immense période de temps, même si l'on ne semble pas y observer de ruptures majeures, notamment dans le peuplement. C'est un peu comme si l'on désignait d'un même terme, sur l'actuel territoire français, une évolution culturelle qui irait du magdalénien à la période dite gauloise. Il n'est donc pas surprenant qu'aussi bien dans leurs productions matérielles que dans leurs organisations sociales et économiques sous-jacentes, les sociétés Jōmon aient suivi une longue trajectoire, depuis de petits groupes de chasseurs-cueilleurs peut-être en partie mobiles, avec des poteries aux formes simples et fort peu décorées, jusqu'aux grands villages permanents, aux monuments mégalithiques et aux poteries surchargées du Jōmon moyen et récent.

- Laurent Nespoulous. Il est vrai que l'on a tendance à focaliser notre attention ou celle du grand public sur la poterie et son ancienneté au Japon - mais en réalité partout en Asie orientale, du Sud de la Chine à la Sibérie -, en oubliant $d^{\prime}$ indiquer que les sociétés de chasseurs-cueilleurs qui les produisent se sont aussi très tôt engagées sur le chemin de la sédentarisation. Jōmon et les sociétés de la fin du Tardiglaciaire et du début de l'Holocène en Asie orientale ne sont peut-être pas le théâtre d'une révolution néolithique, au sens où nous l'entendons en Europe, mais elles connaissent la révolution que constitue la sédentarisation, laquelle a nécessairement des conséquences sur les façons de se représenter le monde.

- Jean-Paul Demoule. De ce point de vue, le développement stylistique de la céramique Jōmon est cohérent. J'avais pu, lors de ma rencontre avec le grand archéologue japonais Makoto Sahara, alors directeur général du Musée national d'histoire et d'ethnologie de Sakura, observer comment, dans le cadre d'une archéologie expérimentale, il reconstituait les différents types d'outils en corde et les différentes manières de les utiliser, selon la typologie développée ici par Inada Takashi.

Qu'exprime plus particulièrement cette variété spatiale et temporelle des styles? Dans l'espace, elle témoigne, lorsque l'on cartographie ces différences typologiques, de la taille de chaque communauté régionale qui exprime ainsi son identité, en se différenciant de ses voisines. Une recherche collective sur le Néolithique grec à laquelle j'ai participé 9 a montré par l'analyse chimique des argiles que, sur chaque site, au sein d'une même entité culturelle régionale, un tiers au moins des récipients les plus ornés, donc les plus valorisés socialement, provenait d'un autre site, que ce soit le récipient lui-même, ou bien son contenu, qui ait été le but de l'échange. Cette circulation permanente explique comment, dans ces sociétés, s'établissait l'homogénéité stylistique des productions céramiques au sein d'un même groupe ethnique s'identifiant comme tel. On peut penser d'ailleurs que cette homogénéité portait aussi sur des

11. Objet en bois spatuliforme à décor entrelacé (sansamon), Hachinohe (département d'Aomori), site de Korekawa-Nakai, Comité éducatif de la ville de Hachinohe. 


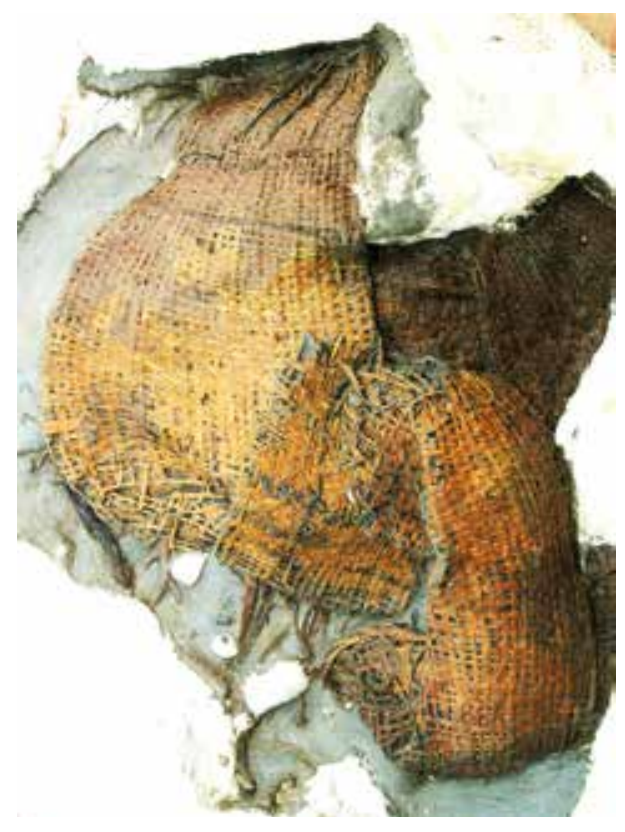

12. Panier (vannerie) mis au jour dans une fosse de stockage, phase archaïque, Saga (département de Saga), site de Higashimyō, Comité éducatif de la ville de Saga.

objets qui ne nous ont pas été conservés, car en matières périssables - comme nous l'indique l'ethnographie.

Dans le temps, l'évolution des types de formes et de décors traduit la pesanteur, ou non, des normes décoratives. C'est souvent à l'échelle d'une génération que ces normes restent stables, pour céder la place à des innovations, là où elles sont autorisées, innovations qui, comme l'ont montré certaines études ethnoarchéologiques ${ }^{10}$, proviennent souvent des marges de la société, moins conservatrices. Il existe aussi des alternances sur le long terme. Ainsi, le Néolithique européen voit alterner des périodes qui favorisent les formes arrondies et les décors curvilignes, et d'autres où I'on préfère les formes anguleuses et les décors de même. Une telle alternance peut être également observée, sur le long terme, dans les deux derniers millénaires de l'histoire de l'architecture européenne. Pour revenir au Jōmon, il serait donc précieux d'analyser dans le détail au fil du temps le rythme et la vitesse des évolutions stylistiques dans chaque région culturelle.

- Laurent Nespoulous. Puisque nous venons d'évoquer la question des ensembles qui se forment, stylistiquement, tout au long du Jōmon, en fonction des régions, j'aimerais aborder l'épineuse question de l'interprétation de ces motifs et de ces formes. On voit très régulièrement, lors des expositions, des tentatives de lecture de l'univers mental des sociétés qui produisent cet « art ». Les figurines, les $\operatorname{dogū}$, tout particulièrement, font l'objet d'une grande attention, autant de certains chercheurs que du grand public. L'essentiel de la production des figurines relève de représentations anthropomorphes aux caractères féminins souvent très poussés. "Vénus ", " déesse " ou " déesse-mère ", figurine ayant vocation à conjurer les maladies ou les dangers liés à la maternité... il y a une palette fort large d'hypothèses. Je dois avouer que je suis toujours un peu mal à l'aise devant ce genre d'approches visant à entrer dans l'univers mental des sociétés anciennes. D'une part, les sociétés du Jōmon, toutes sédentaires soient-elles, sont globalement demeurées des sociétés de chasseurs-cueilleurs, d'autre part, en plus de dix mille ans d'existence, ces sociétés auraient eu plusieurs fois l'occasion de changer, si ce n'est donc pas forcément le geste technique de production de ces motifs et formes, le sens qui leur était donné. Cela suscite chez moi deux questions : comment peut-on travailler sur le sens symbolique des décors de ces objets sans perdre de vue que la profondeur historique qui nous sépare de ces sociétés va nécessairement nous conduire à commettre des interprétations qui en disent plus sur nous que sur les sociétés préhistoriques « non néolithiques »du Tardiglaciaire et de l'Holocène et comment est-ce que vous jugez ce qui est généralement fait pour « romantiser » la Préhistoire Jōmon, au Japon, auprès du grand public ?

- Inada Takashi. Pour rebondir sur la question des fondements économiques de la société Jōmon, nos connaissances ont beaucoup évolué depuis les années 2000. On savait déjà, grâce à la découverte de traces de fabacées (soja, Glycine max et azuki, Vigna angularis var. angularis) à la surface et dans la pâte des poteries, que ces dernières étaient connues des gens du Jōmon. Les recherches en tracéologie d'Obata Hiroki I'on conduit à soutenir que la culture d'une variété locale de soja et d'azuki avait démarré au Jōmon ancien, que la taille 
des grains, dans les régions du Chūbu ou du Kantō (Centre Est de l'archipel), avaient connu une augmentation laissant penser à une domestication, pour enfin se diffuser jusqu'à Kyūshū à I'horizon chronologique Jōmon récent-final. Par ailleurs, on retrouve les traces éparses d'un coléoptère ravageur (le charançon Sitophilus zeamais) qui prospère sur les cultures ${ }^{11}$. La question de la nature de l'économie de la période Jōmon est encore très débattue au Japon. Économie de chasseurs-cueilleurs ? Économie agraire ? Quoi qu'il en soit, la culture de certaines fabacées semble avoir été tout sauf anecdotique.

- Laurent Nespoulous. C'est une « ambiguïté » qui caractérise assez bien la période : on a du stockage, on a sans doute une forme de pratique du jardin potager par endroits, et en même temps on a une activité d'optimisation des ressources naturelles et de prédation sur ces dernières ${ }^{12}$.

- Inada Takashi. Sur la question de l'interprétation des formes et décors produits, je voudrais revenir sur l'une des trois formes d'expression artistique (du beau) indiquées par Jean-Paul Demoule. Il me semble que la deuxième - celle dérivant des objets du quotidien - a particulièrement du sens pour les sociétés préhistoriques. J'ai, par exemple, eu l'occasion de dessiner et analyser les 15 « feuilles de laurier » de Volgu, ces célèbres pointes de l'industrie lithique solutréenne parfaitement symétrique de plus de $30 \mathrm{~cm}$ de long et de moins de $1 \mathrm{~cm}$ d'épaisseur. Il est évident que la technicité des gestes ici allait au-delà du cahier des charges de la production de pointes utilitaires, destinées à être fixée sur une hampe de bois.

Depuis les années 1970, je me suis progressivement mis à penser qu'existait le même type de relation entre la poterie Jōmon et ses décors. Certes, poterie et lithique sont deux industries bien différentes. Kobayashi Tatsuo, qui est la figure tutélaire de la recherche sur le Jōmon de nos jours, a pu dire par le passé que la première était une technique reposant sur un calcul d'addition, là où la seconde relevait, quant à elle, de la soustraction. En effet, autrement dit, dans le cas du travail de la pierre, le sens esthétique procède de la réduction d'un nucléus, alors dans l'autre cas, on procède plutôt par le montage et l'accumulation ensemble de pièces d'argile. Présentés ainsi, on comprend d'autant plus facilement comment les décors de la poterie Jōmon ou les anses surdimensionnées des poteries à motif plastique en flamme (fig. 5) correspondent à un sens esthétique découlant d'objets utilitaires.

Cette distinction entre lithique et poterie peut être à mon sens poussée plus loin, à des distinctions techniques et culturelles propres à la distinction chronologique entre Paléolithique et Néolithique (dans le sens des sociétés humaines avant et après le Tardiglaciaire). Qu'est-ce qui a rendu possible ce « calcul par addition » que l'on retrouve dans la production de poterie ? Vraisemblablement la finesse de la matière travaillée, argile, sable, et des adjuvants de la pâte, semble être un élément important. Les vêtements utilisés au Paléolithique étaient faits de peau de bête, soit par "réduction » de cette dernière. Les sociétés ultérieures, du Néolithique ou du Jōmon, font davantage appel, quant à elles, au tissage ou au tricotage. Il faut pour cela d'abord extraire la fibre des végétaux, et cette dernière est, là aussi, très fine. Partant de là, tout un monde de motifs pourra se développer. Et c'est ainsi qu'il a été possible de retrouver des fibres tricotées datées du Jōmon archaïque lors des fouilles du site de Higashimyō (Kyūshū, département de Saga, fig. 12).

Pour obtenir un objet fonctionnel sur la base de ce calcul de production par addition, il faut, particulièrement quand il s'agit de textile, répéter d'innombrables fois un geste appliqué à une matière extrêmement fine. Le travail de tailleurs chevronnés, comme ils devaient en exister au Paléolithique, était toujours très apprécié, mais l'élément nouveau ici c'est l'importance de gestes simples, réalisables par n'importe qui, comme le piquetage ou le polissage, répétés sans fin et nécessitant surtout de la patience. Néolithique ou Jōmon, 
il me semble que l'on peut dire que la production des objets s'y caractérise alors par le travail de matériaux fins et la répétition de gestes délicats, mais répétitifs et accessibles à tous. On peut sans doute dire de même de la production de l'alimentation, dans le contexte du Néolithique au Proche-Orient, ou dans celui du Jōmon avec la culture des fabacées ou de l'économie de transformation des glands. Au Proche-Orient, au stade dit du Néolithique acéramique, en même temps que commence l'agriculture, on sait déjà polir des haches de pierre, on sait produire des figurines d'argile, des récipients en plâtre de chaux ou de gypse, et on a déjà pris l'habitude d'utiliser l'argile en quantité pour la fabrication des briques en terre sèche des habitations.

Je considère qu'entre le Paléolithique, la phase de transition qui conduit les sociétés humaines à son " après ", que l'on parle de Néolithique ou d'autre chose, l'humanité a d'abord connu un phénomène de révolution d'échelle dans le domaine de l'expertise et de l'activité de production. Nous avons tous (ou presque !) vécu la révolution des nouvelles technologies de la fin du XXe siècle. Les activités de production se sont mises alors à dépendre du circuit intégré et de la numérisation (en valeurs binaires 0 ou 1, donc) de ces tâches. Le Néolithique, à sa manière, a donc été une réduction, encore visible à l'œil nu, de l'échelle de la production, avec des conséquences sur la diffusion de l'information, des arts et des rites matérialisant l'univers mental des sociétés.

Pour revenir à ce que disait Jean-Paul Demoule et à son deuxième type d'expression du beau, les premières formes de décors de la poterie Jōmon sont indissociables de la fonction d'un récipient en terre. Les dogū, les figurines en terre cuite représentant des femmes, si elles existent en petit nombre dès le Jōmon archaïque, connaissent surtout leur âge d'or à partir de la seconde moitié du Jōmon. C'est dans ce contexte que l'on trouve des productions comme les vases à bec verseur ou les poteries en forme de brûle-parfum, fruits d'une diversification formelle. Ces derniers objets présentent une grande diversité tout en

13. Poterie à décor de chasse, phase récente, Hachinohe (département d'Aomori), site de Nirakubo, musée départemental d'Aomori.

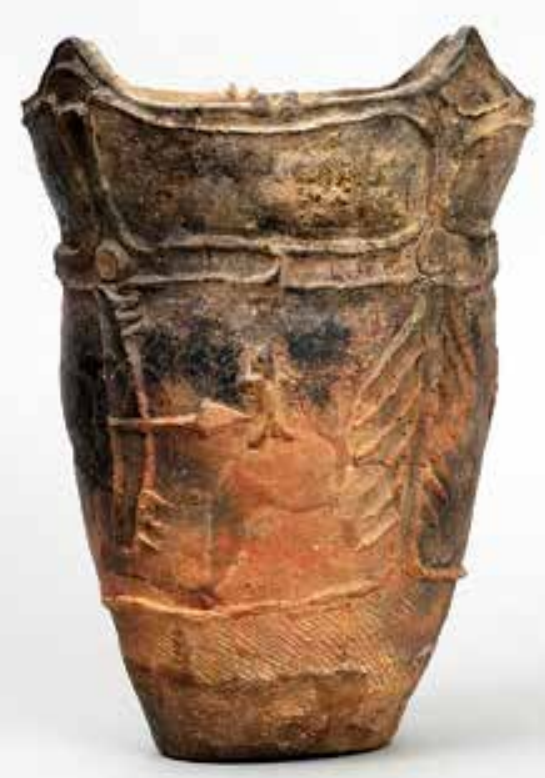
partageant leurs motifs avec ceux des dogū, et il est donc raisonnable de les considérer comme formant un ensemble avec les figurines, répondant à l'univers mental et rituel des sociétés Jōmon à ce moment de leur histoire. Une première alors dans l'expression de cet univers intérieur au moyen de la terre. Les motifs linéaires en incision, qui connaissent un grand développement dans la seconde moitié du Jōmon, sont appliqués à ces nouveaux types de poteries et à ces figurines, mais également à la pierre, au bois de cerf, au bois, et ce pour façonner des objets dont on ne peut percevoir la fonction avec le seul filtre interprétatif de leur utilité / inutilité.

J'ai abordé les questions de la genèse et du développement des décors de la poterie Jōmon dans la mesure où elles conditionnent la compréhension que l'on peut avoir de l'absence de motifs figuratifs incisés, autre particularité évidente de l'art à cette période. En effet, pourquoi, dans le processus de production des objets en terre du Jōmon, alors que les motifs sont appliqués à main levée, les fabricants ne se sont-ils jamais lancés à dessiner à plat le réel ? Par là, j'entends parler de plusieurs choses : la représentation en deux dimensions des éléments existants dans la réalité, tels que des arbres ou que des personnages, dont j'exclue 


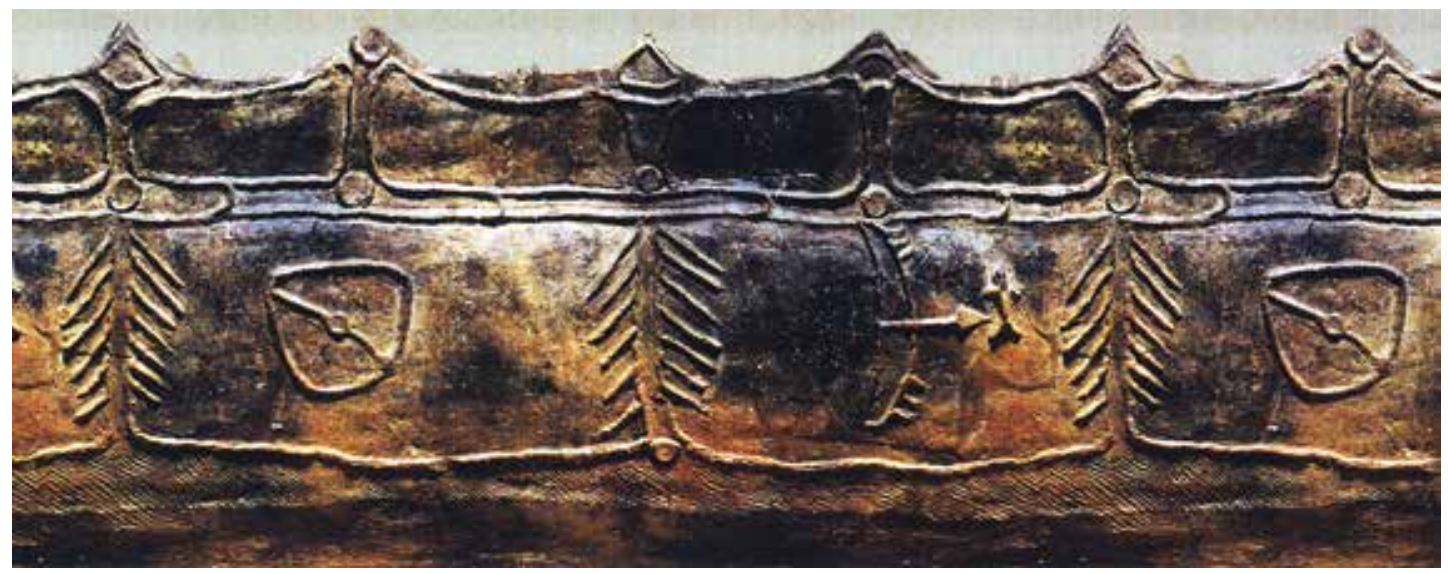

14. Poterie à décor de chasse, phase récente, Hachinohe (département d'Aomori), site de Nirakubo, musée départemental d'Aomori. les figurines et les ajouts plastiques figurants des visages sur certains récipients et tous ces motifs non explicitement figuratifs à nos yeux dont le Jōmon est si riche.

Le Paléolithique en Europe est riche d'un art rupestre que l'on pourrait qualifier de pionnier, mais les sociétés qui s'inscrivent dans des horizons chronologiques ultérieurs, dont celles du Néolithique, installent un rapport

entre le dessin et la formation des premières écritures. C'est le cas des hiéroglyphes en Égypte, de l'écriture cunéiforme en Mésopotamie, ou des premiers idéogrammes en Chine. Pour que se développent ces systèmes d'écriture, il a d'abord fallu en général que se mettent en place des sociétés à forts pouvoirs religieux ou royal, avec des systèmes de gestion des échanges à longue distance relativement avancés. II fallait également que ces sociétés possèdent la capacité d'abstraction permettant de transposer en deux dimensions ce qui existe en trois dans la réalité. De ce point de vue, les sociétés de la seconde moitié du Jōmon évoluaient dans une toute autre direction que certaines sociétés et civilisations contemporaines ailleurs dans le monde. D'ailleurs, plutôt que de dire que Jōmon est en cela particulier, on pourrait dire l'inverse, et que ce sont en fait les sociétés dans lesquelles furent inventés les premiers systèmes d'écriture qui étaient exceptionnelles, ces inventions, ces évolutions se « contentant » par la suite d'essaimer.

Dans le Jōmon récent du Nord-Est de l'archipel, on voit apparaître un nouveau type de motif dit de chasse (shuryō-mon), mettant en scène, sur la panse d'un récipient, un arc, une flèche encochée et une proie (fig. 13 et 14). Le motif est réalisé par application de cordon de terre, et est situé à l'intérieur d'une cellule rectangulaire qu'il vient donc rehausser. Dans un autre registre mais toujours le même contexte, le cercle de pierres dressées d'Ōyu (département d'Akita), a livré de petites plaques rectangulaires de terre cuite, sur lesquelles figure chaque fois un personnage, matérialisé à l'aide de perforations (1 à 6 par partie du corps) représentant la bouche, les yeux, le ventre et les épaules d'un individu (fig. 15). Si ces exemples de représentations figuratives demeurent toutefois très rares, ils apportent au moins une indication sur l'existence de la capacité à les produire chez les habitants de l'archipel à cette période. S'ils ne se généralisèrent jamais, il est donc sans doute plus juste de penser que c'était parce que leur nécessité ne se fit jamais sentir dans le contexte social et culturel du Jōmon.

La production de dogū, représentation en volume du corps humain, n'est pas, d'un point de vue technique, un problème dans une société comme le Jōmon qui maîtrise la transformation de la terre. La vraie question serait plutôt : pourquoi / comment une chose aussi éloignée de 


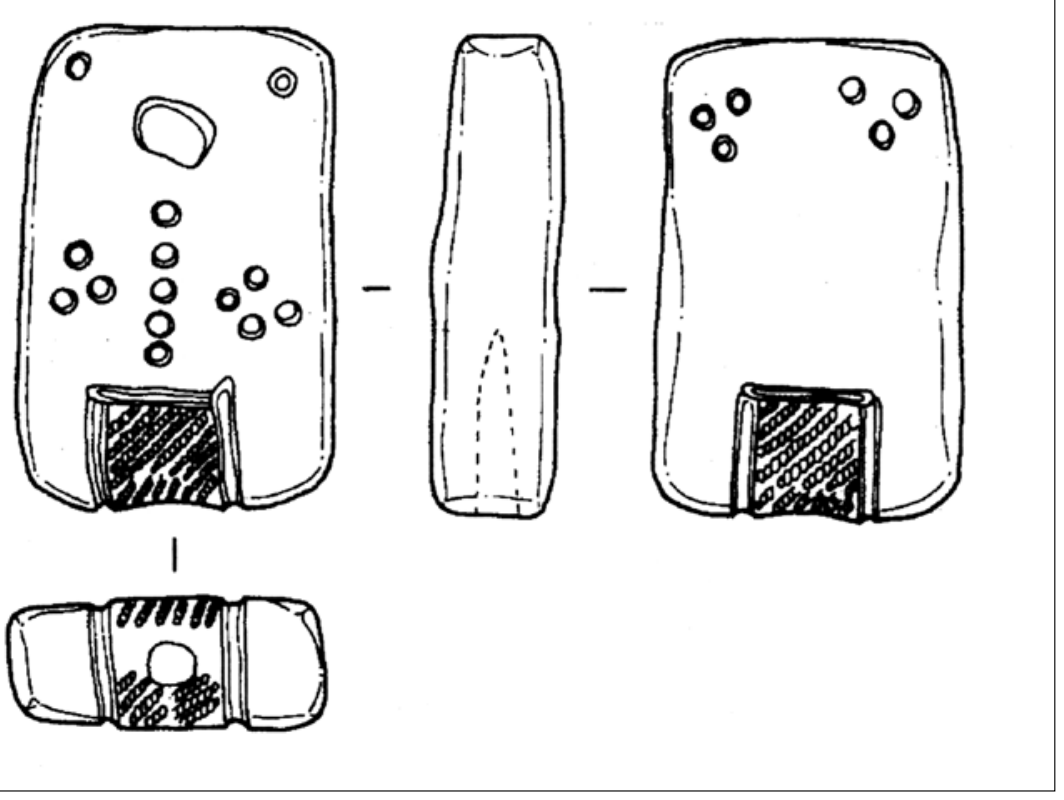

15. Plaquette en terre cuite à perforations, phase récente, Kazuno (département d'Akita), cercle de pierres d'Ōyu, Comité éducatif de la ville de Kazuno. la sphère évidente de l'utile est devenue si répandue ? Il y a d'ailleurs d'autres objets tout aussi « inutiles », sous la forme de plaques de pierre ou de terre. Et c'est donc là, comme le dit Laurent Nespoulous, que toute sorte d'hypothèses - déesse mère, etc. - se font concurrence. On ne peut évidemment pas trancher la chose en quelques mots, mais de nombreuses interprétations se fondent sur des comparaisons ethnographiques avec d'autres sociétés que celles de l'archipel. Il est évidemment important de garder une attitude ouverte à la comparaison, et de dépasser aussi bien les distances géographiques et chronologiques. Il est également capital de garder à l'esprit les limites de ce type d'exercice, et de bien mesurer à quel point ils rejoignent ce que I'on sait des caractéristiques chronologiques et régionales des dogū. Depuis les années 1990, le travail de classification et de calage chronologique de ces figurines a connu de grandes avancées $^{13}$, et on peut dire que continuer à proposer des théories sur les dogū sans prendre en compte ces dernières études relève surtout de la discussion de comptoir.

Il en va de même pour ce qui est d'écrire un « roman » autour du Jōmon. On a pu parler par le passé de « civilisation Jōmon », le terme de civilisation rejoignant ici son sens prestigieux le plus commun. Il est bien possible que si les chercheurs s'enferment dans leur bulle académique de préhistorien, les sociétés anciennes de l'archipel soient alors monopolisées dans la construction d'un nationalisme plein d'illusions de grandeur. Et c'est bien pour cela que les efforts consentis ces dernières années pour maintenir un dialogue entre les archéologues et le public, qu'il s'agisse de transmission des savoirs ou de valorisation du patrimoine archéologique, sont si importants.

- Jean-Paul Demoule. La question de l'interprétation des représentations des sociétés anciennes sans écriture est évidemment complexe. André Leroi-Gourhan s'était moqué, dans son petit livre sur Les religions de la préhistoire (1964) des diverses interprétations qui ont jalonné les études sur l'art préhistorique occidental. Son hypothèse d'une dualité masculin / féminin 
redoublée, sur les parois des grottes, par l'opposition entre le cheval d'une part, et l'auroch ou le bison de l'autre, et renforcée par l'association entre ces animaux et des représentations stylisées de sexes masculins ou féminins, reste relativement convaincante, même si elle s'applique surtout à la période magdalénienne, et pas du tout à l'aurignacien, tel qu'on peut le voir dans la grotte Chauvet, il y 36000 ans. Ces sociétés occidentales de chasseurs-cueilleurs représentaient surtout des animaux car elles se pensaient comme une espèce animale parmi d'autres, et pas la plus dangereuse, et pratiquaient sans doute le totémisme, où chaque groupe descend d'un ancêtre mythique, souvent un animal.

Mais quand elles ont représenté des humains, ce fut en grande majorité des humaines, aux caractères sexuels exagérés. Et c'est aussi le cas dans beaucoup d'autres sociétés humaines de par le monde. On a voulu y voir traditionnellement des symboles de fécondité, voire des " déesses mères ». J'aurais tendance à y voir plutôt des préoccupations autour de la sexualité, chez la seule espèce de mammifères où les cycles féminins ne rendent pas à intervalles réguliers l'accouplement impossible, comme l'avait déjà remarqué en son temps l'ethnologue Bronisław Malinowski ${ }^{14}$. Le fait que les relations sexuelles puissent être pratiquées en permanence a pour conséquence des tensions sociales permanentes, comme nous le démontrent les plus anciens textes connus, chargés d'infractions sexuelles et d'enlèvements de femmes. De ce point de vue, les figurines féminines du Jōmon, les dogū, même si elles sont peu sexualisées, pourraient être interprétées en ce sens, sans qu'on puisse aller beaucoup plus loin.

Quant à l'idée d'une grande déesse mère originelle, on ne peut qu'y être réticent, car dans toutes les sociétés humaines connues le pouvoir appartient aux hommes, même si cette domination est plus ou moins brutale selon les cas. Certes, au Japon, Amaterasu est la déesse du soleil et ancêtre des empereurs, mais elle n'est pas une divinité suprême, pas plus que les déesses d'autres panthéons polythéistes, que ce soit en Inde ou en Grèce, par exemple. Et elle n'est évidemment connue que lorsque nous disposons de textes, et donc dans le contexte de sociétés étatiques. L'idée d'une déesse-mère originelle est un peu un anachronisme, le retournement du grand dieu masculin des religions monothéistes, formes religieuses très récentes, qui n'apparaissent que dans les tout derniers siècles avant notre ère, au plus tôt. C'est pourquoi les travaux de l'archéologue lithuano-américaine Marija Gimbutas, même si elle est très respectée dans les milieux féministes nord-américains, n'emportent pas la conviction ${ }^{15}$. On pourrait tout aussi bien interpréter ces figurines féminines comme un regard masculin sur la sexualité. Et l'on sait par ailleurs que l'une des préoccupations principales des religions historiques est le contrôle de la sexualité féminine.

Quant à la seconde question posée par Laurent Nespoulous, je suis évidemment beaucoup moins bien placé que Inada Takashi pour en traiter. On doit noter cependant que le Japon est le pays qui, au monde, consacre le plus d'argent à l'archéologie préventive par rapport au nombre d'habitants, même si ces efforts ont diminué avec la politique libérale du gouvernement actuel. Cet intérêt des citoyens pour l'archéologie est illustré par le nombre et la qualité pédagogiques des musées archéologiques japonais, avec leurs reconstitutions en plein air, leurs écrans interactifs et les efforts de conservation in situ des sites archéologiques. De fait, les visiteurs y viennent en grand nombre. Le soutien des pouvoirs publics à l'archéologie peut être ambigu, lorsqu'elle est détournée pour servir le « roman national », comme cela est le cas dans beaucoup de pays, France comprise. C'est pourquoi les archéologues doivent être à chaque fois très vigilants pour empêcher de tels détournements et combattre les mythes historiques éloignés de la réalité. Ce n'est pas parce que les Français d'aujourd'hui descendraient des Gaulois ou des Francs qu'ils sont Français, pas plus que les Japonais d'aujourd'hui seraient Japonais parce qu'ils descendraient des sociétés Jōmon puis Yayoï. Ce qui définit une nation, c'est la volonté de ses citoyens de vivre ensemble, c'est « un plébiscite de chaque jour », comme le disait l'historien Ernest Renan. 
La civilisation Jōmon n'en reste pas moins fascinante, pour avoir produit l'un des plus anciens arts céramiques connus et conçu un mode économique original, à la fois de chasse, de cueillette et de pêche, mais aussi de sylviculture et d'horticulture, un défi aux classifications historiques traditionnelles en Occident. Et bien que ce même nom de " Jōmon » recouvre des réalités sociales, économiques, et sans doute idéologiques fort différentes entre le début et la fin, douze millénaires plus tard, de ce phénomène, les Japonais d'aujourd'hui peuvent être heureux d'en avoir directement sous les yeux les vestiges - à condition de savoir les préserver pour les générations futures.

La contribution d'Inada Takashi a été traduite du japonais par Laurent Nespoulous. 


\section{Jean-Paul Demoule}

Jean-Paul Demoule est professeur émérite de protohistoire européenne, membre honoraire de I'Institut universitaire de France et ancien président de I'INRAP. Il a mené des fouilles en France, en Grèce et en Bulgarie et s'est spécialisé dans l'étude du Néolithique et de l'âge du fer, ainsi que sur l'histoire et le rôle social de l'archéologie. Il a publié, seul ou en collaboration, une trentaine d'ouvrages dont, parmi les plus récents : Naissance de la figure - L'art du Paléolithique à I'âge du fer (Paris, Gallimard, 2007 et 2017), Les dix millénaires qui ont fait l'histoire - Quand on inventa l'agriculture, les chefs et la guerre (Paris, Fayard, 2017), Une histoire des civilisations - Comment l'archéologie bouleverse nos connaissances (Paris, INRAP / La découverte, 2018, en codirection avec D. Garcia et A. Schnapp), Aux origines, l'archéologie - Une science au cœur des grands débats de notre temps (Paris, La découverte, 2020).

\section{Inada Takashi}

Inada Takashi est professeur émérite de I'Université d'Okayama, au Japon, et ancien président de l'Association pour la recherche sur le Paléolithique au Japon. Il a publié de nombreux ouvrages et articles relatifs au Paléolithique, à la période Jōmon et aux antiquités du Japon, ainsi que sur le système administratif de protection des sites archéologiques : "Jōmon bunka no keisei » [Formation de la culture Jōmon], dans Nihon kōkogaku [L'archéologie au Japon], vol. 6, 1986 ; Yūdōsuru kyūsekkijin [Les nomades du Paléolithique] (Tōkyō, Iwanami Shoten, 2001), Kyūsekkijidai [Le Paléolithique au Japon] (Tōkyō, Aoki Shoten, 2010, avec H. Satō) ; Nihon to Furansu no iseki hogo [La protection des sites archéologiques au Japon et en France] (Tōkyō, Iwanami Shoten, 2014); "Bifacial Reduction Sequences Observed on the Solutrean Large "Laurel Leaves" from Volgu ", dans $B S P F$, n ${ }^{\circ} 113-3,2016$; "Les structures en creux et les fosses pièges au Japon du Paléolithique à la fin de la période Jōmon. Un bilan des connaissances actuelles ", avec C. Cupillard, dans N. Achard-Corompt, E. Ghesquière et V. Riquier (dir.), Creuser au Mésolithique (Paris, Société préhistorique française, 2017).

\section{Laurent Nespoulous}

Laurent Nespoulous est maître de conférences à I'INALCO. Il a mené des fouilles au Japon et s'est spécialisé dans les trajectoires que les sociétés de l'archipel japonais ont empruntées depuis la formation des sociétés agraires jusqu'à l'émergence de l'État. Il a également pour centre $d^{\prime}$ 'intérêt les rapports entre archéologie, société et patrimoine, ainsi que l'histoire même de la discipline archéologique au Japon. Il est l'auteur de nombreux articles sur toutes ces questions, et a récemment codirigé Patrimonialisation et identités en Asie orientale (Ebisu. Études japonaises, $n^{\circ} 52,2015$, avec Christophe Marquet et Arnaud Nanta), et participé à Une histoire des civilisations - Comment l'archéologie bouleverse nos connaissances (Paris, INRAP / La découverte, 2018, sous la direction de J.-P. Demoule, D. Garcia et A. Schnapp).

\section{NOTES}

1. Laurent Nespoulous, "Plus de 10000 ans d'histoire des sociétés de chasseurs-cueilleurs ", dans Harada Masayuki, Shinagawa Yoshiya (dir.), Jōmon : Naissance de l'art dans le Japon préhistorique, cat. exp. (Paris, Maison de la culture du Japon à Paris, 2018), Paris, Maison de la culture du Japon à Paris, 2018, p. 36-47.

2. Shinagawa Yoshiya, "La beauté platique des poteries Jōmon », dans Harada, Shinagawa, 2018, cité n. 1, p. 18-23.

3. Nakaya Jiujirō, Nihon sekki jidai teiyō [Précis sur l'âge de la pierre au Japon], Tōkyō, Oka shoin, 1929.

4. Laurent Nespoulous, "Un préhistorien japonais à Paris : Nakaya Jiujirō (1929-1932) », dans Ebisu. Études japonaises, no 51, 2014, p. 99-136.

5. Franz Boas, Primitive Art, Oslo / Paris / Cambridge (Mass.), H. Aschehoug / H. Champion / Harvard University Press, 1927.

6. Franz Boas, L'Art primitif (1927), Marie Mauzé (présentation), Catherine Fraixe et Manuel Benguigui (trad. fra.), Paris, Adam Biro, 2003.

7. Kobayashi Tatsuo, Sōran Jōmon-doki [La poterie Jōmon, une classification générale], Kankōi'inkai, 2008.

8. Yananouchi Sugao, Nihon senshi doki no Jōmon [Jōmon, poterie du Japon préhistorique], Senshi kōkogakkai, 1979.

9. Gerwulf Schneider, Heinz Knoll, Kostas Galis, Jean-Paul Demoule, "Transition entre les cultures néolithiques de Sesklo et de Dimini : Recherches minéralogiques, chimiques et technologiques sur les céramiques et les argiles ", dans Bulletin de Correspondance Hellénique, no 115-1, 1991, p. 1-64 ; Jean-Paul Demoule, "Archéologie, style et société », dans Bruno Martinelli (dir.), L'interrogation du style, Anthropologie, technique et esthétique, Aix-en-Provence, Publications de l'université de Provence, 2005, p. 49-65.

10. Dorothy Koster Wasburn (dir.), Structure and Cognition in Art, Cambridge, Cambridge University Press, 1983.

11. Obata Hiroki, Tane o maku Jōmonjin [Les semeurs de graines du Jōmon], Tōkyō, Yoshikawa kōbunkan, 2016.

12. Laurent Nespoulous, "Le contre-exemple Jōmon », dans Jean-Paul Demoule (dir.), La Révolution néolithique dans le monde, Paris, CNRS Éditions, 2010, p. 19-32 ; Laurent Nespoulous, "Les fosses-pièges du site de Tama New Town au Japon. Une approche du Jōmon des forêts ", dans RAE - Revue archéologique 
de l'Est. Chasse, culte ou artisanat? Les fosses "à profil en $N-V-W$ »,33e supplément, Dijon, 2013, p. 283-293 ; Inada Takashi, "Les structures en creux et les fosses pièges au Japon du Paléolithique à la fin de la période Jōmon. Un bilan des connaissances actuelles », en collaboration avec Christophe Cupillard, dans Nathalie Achard-Corompt, Emmanuel Ghesquière et Vincent Riquier (dir.), Creuser au Mésolithique, actes de colloque (Châlons-en-Champagne, séance de la société préhistorique française, 2016), Paris, Société préhistorique française, 2017, p. 255-272.

13. Au titre des grandes études marquantes qui ont proposé les premiers vrais calages typo-chronologiques par région, deux entreprises, complémentaires, sont à souligner : Kokuritsu rekishi minzoku hakubutsukan [Museum national d'histoire du Japon], Dogū to sono jōhō [Sur les dogū], Kokuritsu rekishi minzoku hakubutsukan kenkyū hōkoku [rapport de recherche du Museum d'histoire du Japon], n 37, 1992 ; Dogū to sono jōhō kenkyūkai [Société d'étude «Sur les dogū »], Dogū kenkyū no chihei [Les dogū pour champ d'étude], Benseisha, 1997-2000, 4 vol.

14. Bronisław Malinowski, La sexualité et sa répression dans les sociétés primitives, S. Jankélévitch (trad. fra.), Paris, Payot, 1932, p. 98.

15. Marija Gimbutas, Le langage de la déesse, Camille Chaplain et Valérie Morlot-Duhoux (trad. fra.), Paris, Éditions Des Femmes, 2006 ; contra : Alain Testart, La déesse et le grain : trois essais sur les religions néolithiques, Paris, Éditions Errance, 2010, et Jean-Paul Demoule, Naissance de la figure - L'art du Paléolithique à l'âge du fer, Paris, Gallimard, 2017, p. 143-145. 